\title{
Visualisierung harmonischer Prozesse mithilfe des Circular Pitch-Class Space am Beispiel der Tristan-Sequenz
}

Ansgar Jabs, Pascal Rudolph

Der Beitrag greift den circular pitch-class space der Neo-Riemannian Theory auf, um mit Bezug auf Richard Cohn und Dmitri Tymoczko eine Visualisierungsmethode zur Diskussion zu stellen, nach welcher harmonische Prozesse als das Resultat virtueller Stimmführung zwischen Tonklassen verstanden werden. Die epistemologischen Potenziale dieser Visualisierungsform werden anhand der Anfangstakte der Einleitung zu Richard Wagners Tristan und Isolde ausgelotet.

The article expands on the concept of circular pitch-class space as used by Neo-Riemannian theorists Richard Cohn and Dmitri Tymoczko. Using this concept as a method of visualization, it presents harmonic processes as a result of virtual voice-leading between pitch classes. Through a case study of the opening bars to Richard Wagner's introduction to Tristan und Isolde, we examine the epistemological potentiality of such visualizations.

Schlagworte/Keywords: circular pitch-class space; Dmitri Tymoczko; Neo-Riemannian Theory; Richard Cohn; Richard Wagner; Tristan und Isolde; virtual voice-leading; virtuelle Stimmführung; Visualisierung harmonischer Prozesse; visualization of harmonic processes

Im Rahmen der Neo-Riemannian Theory gewinnt die Visualisierung von Harmonik für deren analytisches Verständnis zunehmend an Bedeutung. ${ }^{1}$ Vor allem die Figur des Kreises taucht zur Veranschaulichung harmonischer Prozesse immer wieder auf. ${ }^{2}$ In seinem Buch A Geometry of Music stellt Dmitri Tymoczko Harmonien mithilfe des circular pitchclass space dar, d. h. als Gruppen von Tonklassen (pitch-class sets) innerhalb eines Oktavraums. ${ }^{3}$ Dieses Modell besitzt unseres Erachtens ein hohes, bisher noch nicht vollständig ausgeschöpftes Potenzial zur Visualisierung harmonischer Prozesse. Es basiert auf einer Kreisform, auf deren Kreislinie die zwölf Tonklassen des chromatischen Tonsystems wie die Zahlen einer Uhr angeordnet sind. Die zugehörigen Tonklassen eines Akkords werden in dieser Darstellung durch Punkte markiert. Der Dreiklang C-Dur wird beispielsweise wie in Abbildung 1 dargestellt:

1 Vgl. Rieke 2019.

2 Vgl. Cohns Hexatonic Cycles (2012, 17-19) oder Jack Douthetts Modell des Cube dance (Douthett/Steinbach 1998, 254). Der Kreis stellt eine prominente Denkfigur in Musikwissenschaft und -theorie dar, derer sich vor allem formanalytische Untersuchungen bedienen; vgl. Morgan 2000; Hepokoski/Darcy 2006, 611-614; Taylor 2010 und 2016; Rudolph/Küssner 2018. Vgl. ferner auch Berger 2007, 45-129.

3 Vgl. Tymoczko 2011, 36-40. 


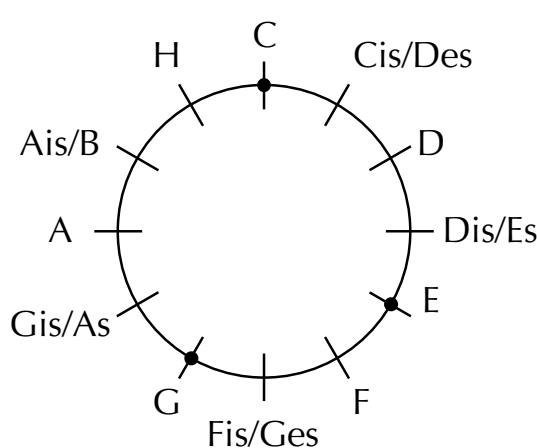

Abbildung 1: C-Dur-Dreiklang, dargestellt im circular pitchclass space ${ }^{4}$

Richard Cohn verwendet in Audacious Euphony ebenfalls diese Darstellungsweise, verbindet die Akkordtöne jedoch zusätzlich mithilfe von Linien untereinander. Die Länge einer daraus resultierenden Kreissehne bildet ein virtuelles Intervall ab, welches zwischen den Tonklassen innerhalb desselben Oktavraums besteht (Abb. 2). Durch diese Kreissehnen entsteht eine geometrische Figur innerhalb des Kreises, welche nicht nur die Intervallstruktur eines Akkords sins Bild setztı, sondern auch eine schnelle Identifikation von Symmetrien ermöglicht (so weist beispielsweise ein gleichseitiges Dreieck auf die gleichen Intervalle zwischen den Tonklassen eines übermäßigen Dreiklangs hin). ${ }^{5}$ Die Intervallstruktur stellt sich bei allen Akkorden eines Typs, beispielsweise des Dur-Dreiklangs, gleich dar und rotiert bei Transposition nur innerhalb des Kreises bei ansonsten gleichbleibender Gestalt.

a)

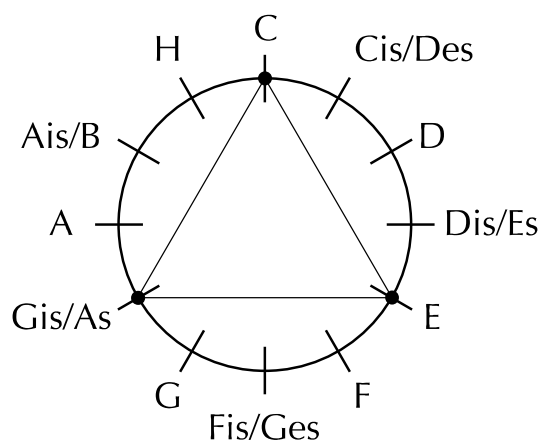

b)

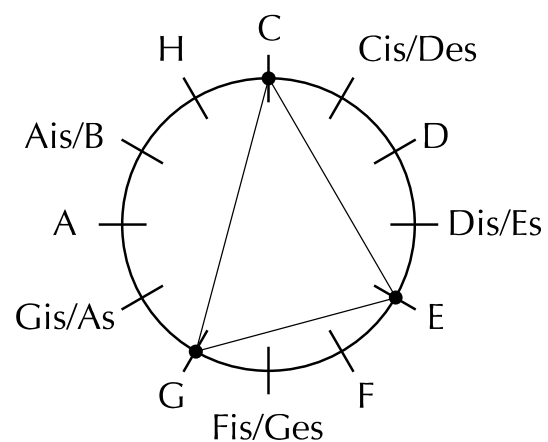

Abbildung 2: a. übermäßiger Dreiklang über c; b. C-Dur-Dreiklang ${ }^{6}$

Wie jede Form der Visualisierung freilich Implikationen enthält und mithin Auskunft darüber gibt, wie ein Konzept in einer zugrunde liegenden Theorie gedacht wird, fällt hier zunächst auf, dass die Visualisierung des circular pitch-class space mit einem System arbeitet, in dem alle zwölf Tonklassen gleichwertig dargestellt werden und enharmonisch äquivalent sind. Demzufolge werden Intervalle, die den gleichen Abstand zwischen zwei Tonklassen aufweisen, identisch dargestellt (z. B. übermäßige Sekunde und kleine Terz). Eine Zugehörigkeit von Tonklassen zu einer bestimmten Tonalität oder deren unterschiedliche Funktionen innerhalb einer solchen werden - anders etwa als in der traditio-

4 Nach ebd., 37.

5 Solche Symmetrien dienen Cohn zur Ableitung verschiedener Akkordstrukturen. So versteht er z. B. den Dur-Dreiklang als Ableitung vom übermäßigen Dreiklang »via single semitonal displacement« (Cohn 2012, 35; vgl. Abb. 2).

6 Nach ebd., 35. 
nellen Funktionstheorie - nicht abgebildet. Weiterhin ist durch die Reduktion der Harmonie auf ihre Tonklassen nicht mehr ersichtlich, ob ein Akkord in Grundstellung oder einer Umkehrung erklingt. Überhaupt entfällt jede Hierarchisierung der am Akkord beteiligten Töne aufgrund ihrer Lage oder Funktion, da die Visualisierung die Identifikation eines Basstons oder gar Grundtons schlechthin verwehrt. In der Darstellung sind also alle beteiligten Tonklassen von gleicher Wertigkeit: Sie sind gleichermaßen an der virtuellen Intervallstruktur beteiligt, die dem Akkord zugrunde liegt.

Tymoczko und Cohn nutzen diese Kreisdarstellung unter anderem, um Fortschreitungen von Akkorden mithilfe einer virtuellen Stimmführung abzubilden. Die einzelnen Tonklassen eines Akkords werden dabei wie Stimmen eines Satzes behandelt, welche sich von einem Akkord zum nächsten in kleinstmöglichen Intervallabständen verschieben. ${ }^{7}$ Es handelt sich hierbei um eine Abstraktion, da nur von den Tonklassen, dargestellt im Oktavraum, und nicht den tatsächlichen Tonhöhen im Notentext ausgegangen wird. So entsteht eine virtuelle Stimmführung, durch welche die Beziehung zweier Akkorde zueinander anhand ihrer Tonklassen beschreibbar wird (Abb. 3). ${ }^{8}$ Die durch die Darstellung suggerierte Stimmbewegung von einer Tonklasse zu einer anderen muss damit weder einer tatsächlichen Stimme noch einer tatsächlichen Bewegung ebendieser in der realen Stimmführung entsprechen (wenngleich beides natürlich vorliegen kann).
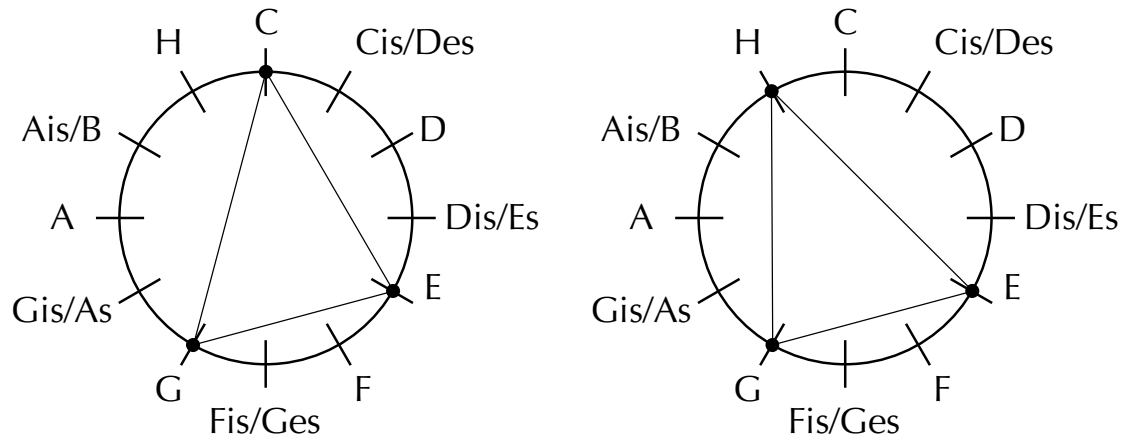

Abbildung 3: Progression vom C-Dur- zum e-Moll-Dreiklang ${ }^{9}$

Hierin lässt sich ein Paradigmenwechsel im Verständnis von Harmonik erkennen: Akkordprogressionen werden nicht als Fortschreitungen der Grundtöne von Funktionen oder Stufen aufgefasst. Stattdessen rückt die Intervallstruktur zwischen den Tonklassen eines Akkords im Verhältnis zu anderen ins Zentrum der Aufmerksamkeit. Das Konzept von Harmonik weist insofern Parallelen zum Kontrapunkt auf, als harmonische Prozesse als Resultat virtueller Stimmführungsprozesse verstanden werden.

7 Vgl. ebd., 33-37 und Tymoczko 2011, 41-44.

8 Auf seiner Internetseite visualisiert Tymoczko die Bewegung von Stimmen zwischen Tonklassen zu Beginn von Frédéric Chopins Prélude e-Moll op. 28/4 mithilfe einer Videoanimation. Mit jedem Akkordwechsel verschieben sich die Töne auf der Kreislinie von einer Tonklasse zur nächsten wie Stimmen in einem Satz. Durch diese Art der Animation kann natürlich die Annahme suggeriert werden, dass der Komposition eine ebensolche Stimmbewegung zugrunde liegt. Ferner färbt Tymoczko die gesamte Darstellung ein, wobei je eine Farbe einem bestimmten Akkordtypus entspricht (Dur-Dreiklang, MollDreiklang, verminderter Septakkord etc.). Damit übernimmt die Farbe die Funktion, die bei Cohn die geometrische Figur innehat, welche durch die Kreissehnen entsteht: Die spezifische Akkordgestalt wird tonklassenunabhängig sofort sichtbar (vgl. https://dmitri.mycpanel.princeton.edu/ChordGeometries.html [15.12.2019]).

$9 \quad$ Nach Cohn 2012, 35. 
Während Tymoczko und Cohn diese Visualisierungsform in erster Linie verwenden, um strukturelle Symmetrien innerhalb eines Akkords sichtbar zu machen und anhand von strukturellen Ähnlichkeiten harmonische Verwandtschaftsverhältnisse zu begründen, ${ }^{10}$ glauben wir, dass sie selbst methodisches Potenzial birgt: Sie gestattet eine neue Perspektive auf harmonisches Geschehen und ermöglicht es, Akkordfortschreitungen vorrangig als die Beziehung zwischen verschiedenen Intervallstrukturen der beteiligten Tonklassen zu verstehen. Daher verwenden wir diese Visualisierung insofern konsequent für die Analyse, als wir - ähnlich wie im Fall einer Akkordsymbolschrift - alle Akkorde der betrachteten harmonischen Passage symbolisch, nämlich als Kreise darstellen. Für einen solchen methodischen Ansatz erachten wir ein paar Modifikationen dieser Visualisierung für sinnvoll. Im Unterschied zu Cohn verbinden wir die Tonklassen durch Linien nicht direkt untereinander, sondern mit dem Kreismittelpunkt, sodass sie wie die Zeiger einer Uhr erscheinen. Auf diese Weise wird die Kreisfläche, die dem Raum einer Oktave entspricht, in einzelne Kreissegmente aufgeteilt, welche analog zu den Kreissehnen bei Cohn die Intervalle zwischen den Tonklassen abbilden. Dadurch erhält jeder Akkordtyp ebenfalls eine spezifische visuelle Gestalt. Der übermäßige Dreiklang entspricht beispielsweise einer Teilung des Kreises in drei gleiche Segmente, der verminderte Septakkord in vier usw. Dies ermöglicht die schnelle Identifikation von symmetrischen Strukturen; Transpositionen von Akkorden sind weiterhin als Rotationen der Gestalt innerhalb des Kreises sichtbar. Die jeweilige Größe der virtuellen Intervalle wird durch die flächenhafte Darstellung etwas deutlicher (Abb. 4).
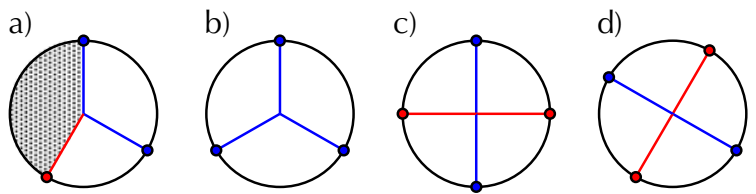
Abbildung 4: Visualisierung verschiedener Dreiklangsstrukturen; a. Dur-Dreiklang, b. übermäßiger Dreiklang, c. und d. Voll- verminderte Septakkorde

Außerdem verwenden wir in unserer Darstellung zwei Farben zur Unterscheidung der zwei Ganztonreihen im Tonklassensystem (Blau: $c$, d, e, fis, gis und ais; Rot: des, es, f, g, $a$ und $h$ ). Dies soll einer besseren Orientierung über die Abstände der Tonklassen in der Kreisform dienen. Bei Fortschreitungen lässt sich so schneller erkennen, ob eine Tonklasse etwa einen Ganztonschritt (der Nachbarton hat die gleiche Farbe) oder einen Halbtonschritt (der Nachbarton hat die jeweils andere Farbe) von einer benachbarten entfernt liegt. Ferner kann auch die Größe der Intervalle zwischen den Tonklassen mithilfe dieser Klassifizierung schneller erfasst werden: Haben zwei Tonklassen dieselbe Farbe, ist das Intervall zwischen ihnen ganztonbasiert (d. h. große Sekunde, große Terz oder Tritonus). Unterscheidet sich die Farbe zweier benachbarter Tonklassen, handelt es sich um ein Intervall, das einen Halbtonschritt enthalten muss (d. h. kleine Sekunde, kleine Terz oder Quarte). Darüber hinaus werden Kreissegmente, die einer Sekunde oder einer Quarte entsprechen, mit einer grauen Schraffur versehen, während die Flächen, die Terzen repräsentieren, weiß bleiben. Wir erachten dies als hilfreich, da die Struktur der meisten Akkorde auf dem Terzschichtungsprinzip beruht und daher die grau schraffierte Sekunde oder Quarte anzeigt, an welcher Stelle mit dieser Struktur gebrochen wird, sodass eine schnellere Orientierung innerhalb eines dargestellten Akkords ermöglicht wird (Abb. 5). Wir verzichten bewusst auf die Beschriftung des Kreises mit Tonnamen. Entscheidend ist 
nicht, welche spezifischen Tonklassen der Akkord enthält, sondern welche Intervallstruktur er besitzt und wie sich diese in der Fortschreitung zum nächsten Akkord wandelt.
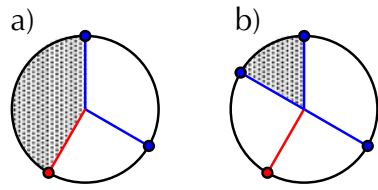

Abbildung 5: a. C-Dur-Dreiklang - Graufärbung der Quarte;

b. kleiner C-Dur-Septakkord - Graufärbung der Sekunde

Welche Potenziale und Grenzen diese Visualisierungsmethode mit sich führt, soll im Folgenden anhand der ersten Takte der Einleitung zu Richard Wagners Tristan und Isolde gezeigt werden (Bsp. 1). ${ }^{11}$

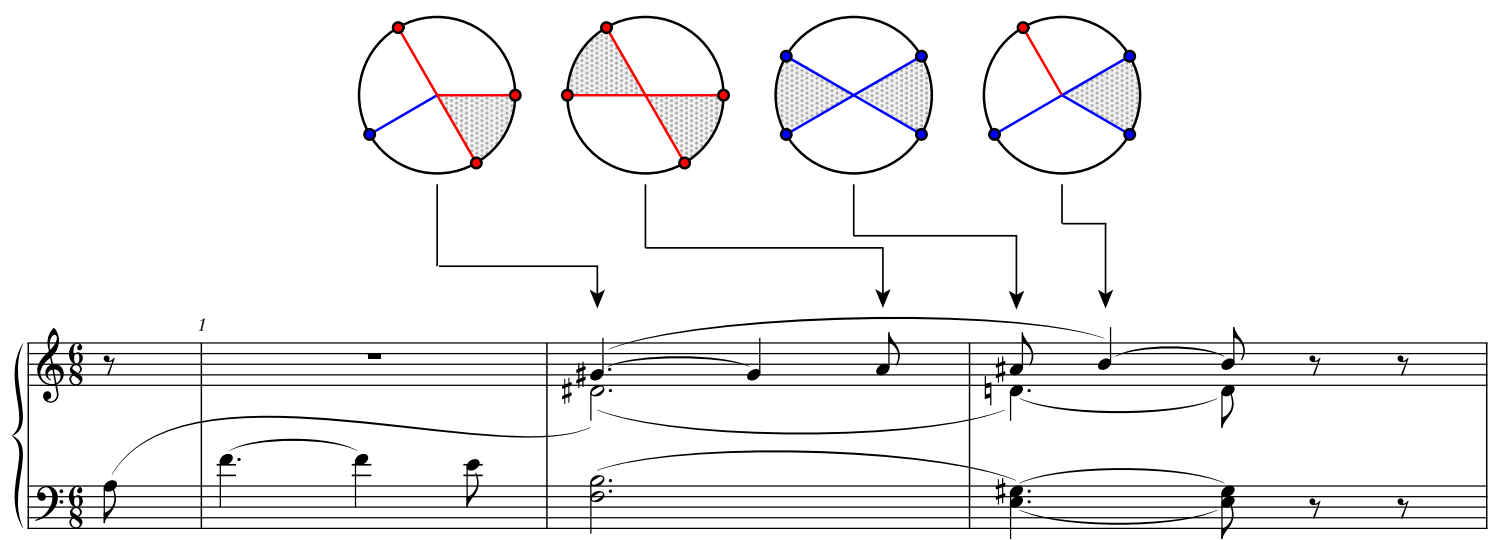

Beispiel 1: Richard Wagner, Einleitung zu Tristan und Isolde, erste harmonische Progression (T. 1-3)

Die Sektoren des ersten Kreises bilden die Intervallstruktur zwischen den Tonklassen des berühmten `Tristan-Akkords` ab, die der eines halbverminderten Septakkords entspricht, ohne einen der Töne zu priorisieren oder die Intervalle in einer Reihenfolge ordnen zu wollen. Es erfolgt also keine Deutung des Akkords hinsichtlich eines Grundtons. ${ }^{12}$ Wie in der Abbildung zu sehen ist, entsteht die Intervallstruktur des zweiten Kreises durch die Veränderung einer einzigen Tonklasse. Der zweite Kreis weist eine symmetrische Intervallstruktur auf (d. h. es lässt sich durch die Visualisierung eine Spiegelachse ziehen), welche der eines French sixth chord-Typus ${ }^{13}$ entspricht. Im dritten Kreis ist zu erkennen, dass der folgende Akkord die identische Struktur aufweist, da auch hier die Kreissektoren zwei gegenüberliegende große Sekunden und zwei gegenüberliegende große Terzen abbilden. Die Gestalt wurde lediglich als Ganzes um einen kleinen Winkel rotiert, der einem Halbton entspricht (vgl. die unterschiedlichen Farben). Wir möchten erneut betonen, dass es sich hierbei nicht um die tatsächliche Stimmführung handelt, sondern um das Verhältnis der pitch-class sets zueinander, aus welchen sich die jeweiligen Akkorde kons-

11 Die Notenbeispiele in den folgenden Abbildungen richten sich nach Voss 2013.

12 Für ebensolche funktionsharmonischen Deutungen vgl. Karsten 1951 und Danuser 1998, 832-838.

13 Dieser Begriff bezieht sich auch im Folgenden nur auf den intervallischen Aufbau aus zwei Sekunden und zwei großen Terzen, nicht auf die Tradition seiner Verwendung und Funktion als übermäßiger Terzquartakkord. 
tituieren. ${ }^{14}$ Bei der Fortschreitung vom dritten zum letzten Kreis wird abermals eine Tonklasse durch eine im Halbtonabstand benachbarte ersetzt, wodurch die Symmetrie der Intervallstruktur eines French sixth chord zugunsten eines Dominantseptakkords verloren geht. Bereits Heinrich Poos machte auf die Symmetrien aufmerksam, die dieser Passage zugrunde liegen, indem er sie als »ein mit Spiegeln versehenes Ideengitter ${ }^{15}$ bezeichnete (Bsp. 2).

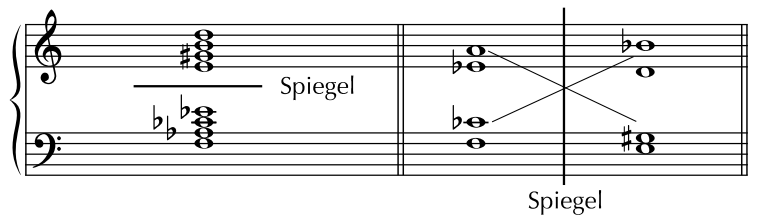

Beispiel 2: Richard Wagner, Einleitung zu Tristan und Isolde, Spiegelungen innerhalb der ersten vier Akkorde ${ }^{16}$

Sowohl der Dominantsept- als auch der halbverminderte Septakkord unterscheiden sich hinsichtlich der Intervallstruktur von einem French sixth chord nur um einen Halbtonschritt, ${ }^{17}$ jedoch stehen sie sich strukturell diametral gegenüber: Basiert der Dominantseptakkord auf einem Dur-Akkord, über dessen höchstem Ton, der Quinte, noch eine kleine Terz hinzugefügt wird, so entsteht der halbverminderte Septakkord, indem man dem Grundton eines Molldreiklangs eine kleine Unterterz hinzufügt (Bsp. 2, links). ${ }^{18}$ Dieser umgekehrte Aufbau in der Intervallstruktur wird in der Kreisabbildung durch die Anordnung der Segmente deutlich: Beide Kreisfiguren enthalten zwei kleine Terzen nebeneinander, die große Terz und die große Sekunde sind jedoch hinsichtlich ihrer Position in der Gestalt vertauscht. Darüber hinaus unterteilt Poos die Akkordfolge mithilfe einer Spiegelachse zwischen den beiden French sixth chords (Bsp. 2, rechts), wodurch sich zwei Akkordpaare ergeben. ${ }^{19}$ Das erste Akkord-Paar wird durch die Klangcharakteristik des halbverminderten Septakkords bestimmt, während im zweiten die Klangcharakteristik des Dominantseptakkords vorherrscht. Die Intervallstruktur des French sixth chord dient sozusagen als tongeschlechtsneutraler Übergang zwischen Anfangs- und Schlussakkord dieser Passage, wobei die Beziehung zwischen den verschiedenen pitch-class sets der beiden Akkord-Paare identisch ist: Während eine Tonklasse durch eine um einen Halbtonschritt benachbarte ersetzt wird, werden alle anderen Tonklassen beibehalten. Durch die Visualisierung werden die verschiedenen in dieser Passage wirksamen Symmetrieverhältnisse in geometrischer Anschaulichkeit wie folgt sins Bild gesetzt (Abb. 6):

- die Spiegelung innerhalb einer Akkordstruktur (der French sixth chord als ssymmetrischer Akkord $)$,

- die Spiegelung innerhalb der gesamten Akkordfolge (der chiastische Aufbau),

14 In der Partitur spielt beispielsweise das erste Fagott an dieser Stelle eine kleine Terz abwärts ( $h$-gis in T. 2-3) statt einer kleinen Sekunde (vgl. Bailey 1985), sodass hier die reale Stimmführung nicht der virtuellen Stimmführung in der Kreisvisualisierung entspricht. Vgl. ebenso die Stimmführung im ersten Fagott (T. 6-7).

15 Poos 1987, 73.

16 Nach ebd.

17 Vgl. dazu Cohns Systematisierung der Dominantseptakkorde und halbverminderten Septakkorde als tristan genus chords (2012, 148-152).

18 Diese Feststellung basiert auf der Theorie des `Dur-Moll-Dualismus`, die von Harrison (1994) für die Neo-Riemannian Theory adaptiert wurde. 
- das Spiegelungsverhältnis zwischen zwei Akkorden hinsichtlich ihrer Intervallstruktur ( sumgekehrter` Aufbau der Intervallstruktur des halbverminderten Septakkords im Vergleich zum Dominantseptakkord).

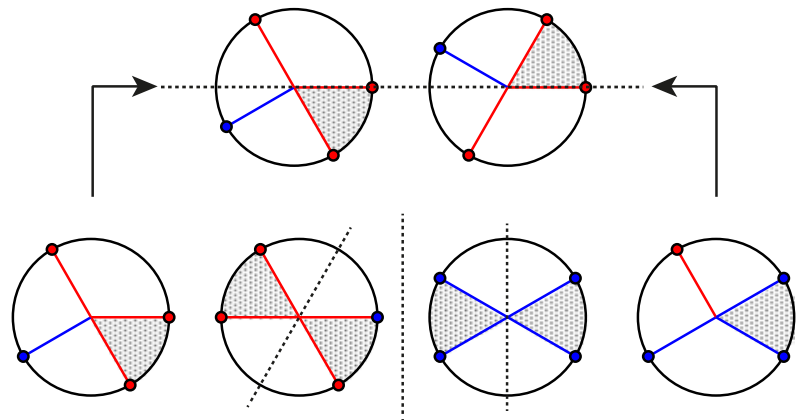

Abbildung 6: Richard Wagner, Einleitung zu Tristan und Isolde, Spiegelungen und Symmetrien innerhalb und zwischen den ersten vier Akkorden. Der obere rechte Kreis entsteht durch die Rotation des vierten Kreises um $30^{\circ}$ (entspricht der Größe eines Halbtons).

Die harmonischen Ereignisse dieser Passage lassen sich mithilfe der Kreisabbildungen als das Resultat virtueller chromatischer Stimmführungsereignisse deuten. Entscheidend ist dabei nicht, wie die Töne im Sinne einer Funktionstheorie zu hierarchisieren sind, sondern wie sich die strukturellen Beziehungen zwischen den Tonklassen, die den Akkorden jeweils zugrunde liegen, zueinander verhalten. Bei einer Ausweitung der Analyse auf die ersten elf Takte der Einleitung offenbart die Visualisierung mehrere Parallelen zur ersten harmonischen Progression (Bsp. 3). Dass es sich etwa in den Takten 6 und 7 um eine reale Sequenz der gesamten Takte 2 und 3 um eine kleine Terz aufwärts handelt, ist durch die Rotation der ersten Zeile um $90^{\circ}$ in der zweiten Zeile bei ansonsten gleichbleibender Gestalt zu erkennen. Die dritte Zeile (T. 10 und 11) lässt sich als Fortsetzung des Sequenzvorgangs verstehen, weist aber nicht nur Ähnlichkeiten, sondern auch einige substanzielle Abweichungen zu den vorangegangenen Abschnitten auf.

Zunächst ist zu sehen, dass der erste Akkord hinsichtlich seiner Intervallstruktur mit dem sTristan-Akkord und seiner Transposition in der zweiten Zeile identisch ist, jedoch eine andere Rotation aufweist. Der erste Akkord ist gegenüber Takt 6 nun nicht nur um weitere $90^{\circ}$, sondern um $180^{\circ}$ rotiert, was dem Intervall eines Tritonus entspricht. Jedoch zeigt sich bei der Fortschreitung vom ersten zum zweiten Kreis zugleich eine erneute Sequenzierung um eine kleine Terz $\left(90^{\circ}\right)$ aufwärts, und zwar in derjenigen Tonklasse, die auch hier durch ihren Nachbarn im Halbtonabstand abgelöst wird. Während also das Prinzip der Kleinterzsequenz in dieser speziellen Beziehung zweier Tonklassen fortgeführt wird, bricht die harmonische Konstellation mit diesem Sequenzintervall. Die Intervallstruktur eines halbverminderten Septakkords als Ausgangspunkt der Progression bleibt gewahrt, ${ }^{20}$ doch befindet sich die charakteristische Beziehung zweier Tonklassen im Halbtonabstand innerhalb der Akkordstruktur an anderer Position als bisher. Hieraus resultiert im zweiten Akkord der Passage nicht mehr die Intervallstruktur eines French sixth chord, sondern die eines Mollseptakkords. Dieser ist in seiner Intervallstruktur ebenfalls symmetrisch angelegt, besitzt allerdings eine völlig andere Klangfarbe, die aus der fehlenden zweiten Sekundreibung resultiert. Mit der Ersetzung der beiden benachbarten Tonklassen durch die dazwischenliegende wird die Intervallstruktur des Mollseptakkords in einen übermäßigen Dreiklang überführt, dessen Intervallstruktur abermals symmetrisch ist.

20 Poos $(1971,541)$ erkennt ebenfalls einen formalen Zusammenhang zwischen den Anfangsakkorden der Sequenzen über deren zugrundeliegende Intervallstruktur als »die vom Sequenzzusammenhang an dieser Stelle geforderte lexikalische Einheit shalbverminderter Septakkord««. 

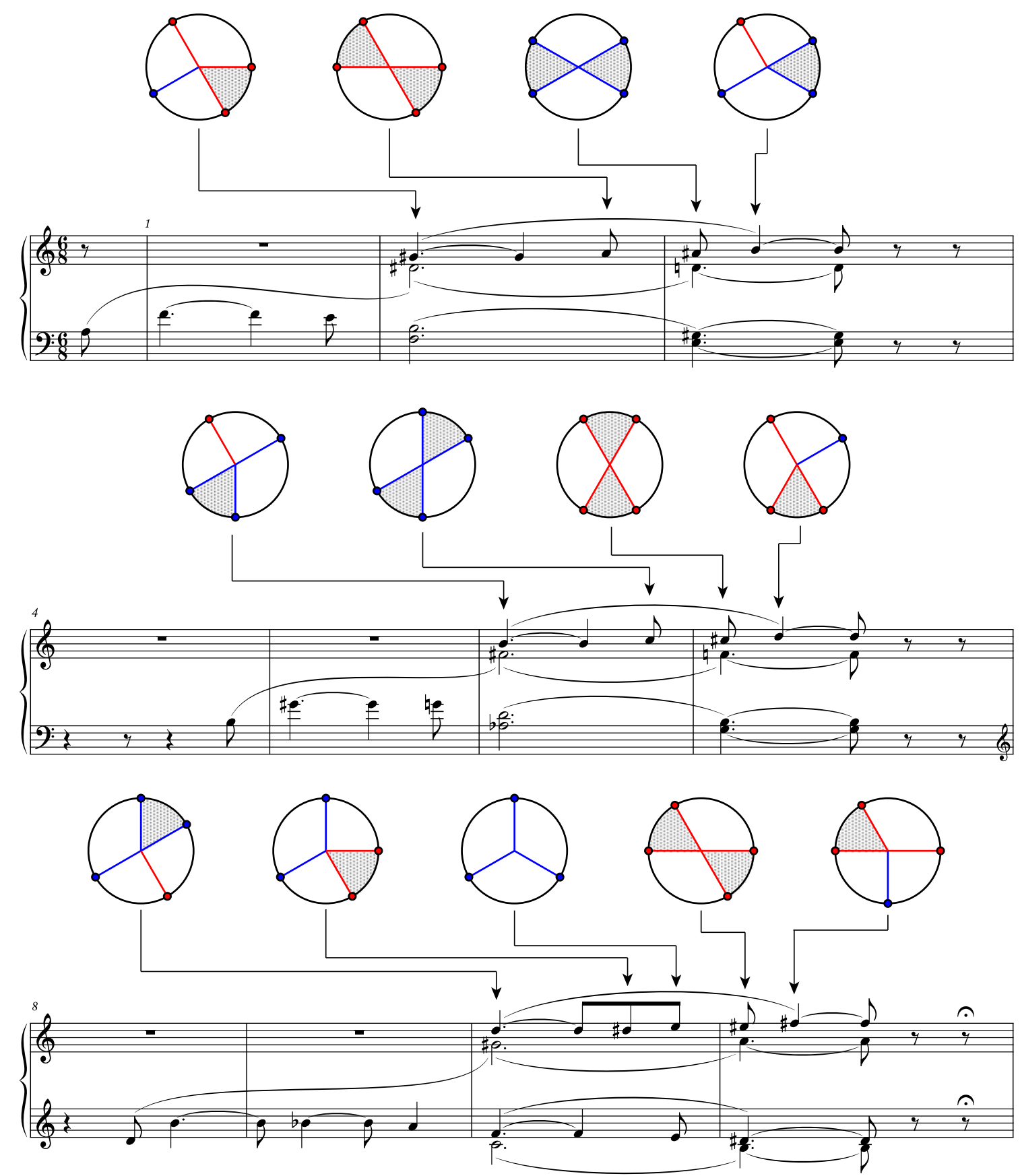

Beispiel 3: Richard Wagner, Einleitung zu Tristan und Isolde, T. 1-11 mit Visualisierung der Akkordstrukturen

Aus diesem entfaltet sich dann (T. 11) die charakteristische Intervallstruktur des French sixth chord, ${ }^{21}$ womit letztlich der Akkord erreicht ist, welcher auch in den beiden vorangegangenen Sequenzgliedern an vorletzter Stelle steht und in gleicher Weise in einen Dominantseptakkord führt. So bilden die beiden Schlussakkorde nun wieder eine reale Sequenz der vorangegangenen Schlussakkorde in den Zeilen 1 und 2 ab, die allerdings im Abstand einer großen Terz vollzogen wird. Erneut finden sich also zu Beginn und am

21 Die Symmetrie der Intervallstrukturen zwischen den Tonklassen sowie der Farbwechsel lassen die Visualisierung optisch wie ein vierstimmiges `Negativ` des vorangegangenen übermäßigen Dreiklangs aussehen. 
Ende eines Abschnitts die Intervallstrukturen des halbverminderten Septakkords und des Dominantseptakkords, und erneut zeichnet sich der Übergang durch Akkorde aus, deren Intervallstruktur interne Symmetrie aufweist. Allerdings wird der Übergang in der dritten Zeile mit einem Mollseptakkord eingeleitet, der in den übermäßigen Dreiklang führt und erst in einem dritten Akkord die Intervallstruktur des French sixth chord als letztes Übergangsglied erreicht. In dieser harmonischen Variation vollzieht sich die Modifikation des Sequenzintervalls zu einer großen Terz. So wird mit den Schlussakkorden eine Quintsequenz der ersten harmonischen Progression erreicht. ${ }^{22}$

Die zweite Sequenzierung der Eingangstakte stellte für viele Forscher ein enigmatisches Gebilde dar. Der mit ihr verknüpfte >dritte Tristan-Akkord ist etwa laut Karsten »harmonisch überhaupt nicht selbständig deutbar «. ${ }^{23}$ Poos sieht in ihm das Resultat motivischer und formkonstituierender Prozesse und bezeichnet ihn als Vorboten der »freie[n] Tonalität", einen »aus dem kadenzharmonischen Zusammenhang emanzipierte[n] monistisch-motivische[n] Akkord «. ${ }^{24}$ Ünlü wiederum deutet ihn als einen bivalenten Akkord, der einerseits »noch Teil des 2. Sequenzglieds mit dem Tonzentrum C-Dur « ist und andererseits bereits »an der Schwelle zum dominantischen E-Dur « ${ }^{25}$ des dritten Sequenzglieds steht. Die Kreisdarstellungen wiederum exponieren - frei von jedweder Grundton- oder Tonzentrumsbestimmung sowie motivisch-/formanalytischer Tradition - zum einen die mit den anderen Eingangsakkorden identische Intervallstruktur zwischen seinen Tonklassen durch die geometrischen Formationen und zum anderen das Sequenzintervall der kleinen Terz durch das identische Bewegungsmuster zwischen den Tonklassen vom ersten zum nächsten Akkord (drei der Tonklassen bleiben erhalten, eine wird durch ihren um einen Halbton höher liegenden Nachbarn ersetzt). Durch diese Visualisierungsmethode wird die Beziehung von Intervallstrukturen zwischen Tonklassen zum zentralen Parameter der Harmonieanalyse. Dadurch lassen sich Zusammenhänge zwischen verschiedenen Passagen erkennen, die im Verhältnis der durch die Tonklassen entstehenden Intervallstrukturen begründet sind. Sicherlich wäre es vermessen, zu glauben, damit die harmonische Rätselhaftigkeit des Tristan-Beginns aufzulösen, welche bereits unzählige analytische Auseinandersetzungen auf den Plan rief. ${ }^{26}$ Vielmehr war es unser Anliegen, anhand eines Beispiels, welches wir bewusst aufgrund seiner Prominenz ausgewählt haben, epistemologische Potenziale dieser Visualisierungsform aufzuzeigen. Wie jede Form der Visualisierung stellt natürlich auch diese eine enorme Reduktion dar, indem sie musikalische Parameter ignoriert, welche durchaus einen Einfluss auf das Verständnis für die Harmonik besitzen (neben dem tonalen Zusammenhang etwa reale Stimmführungsphänomene, Registerfragen, Rhythmik, Instrumentation etc.). Dennoch glauben wir, dass diese Art der Visualisierung einen neuen Blickwinkel auf harmonisches Geschehen ermöglicht, da sie die Beziehungen zwischen den Intervallstrukturen von Akkorden jenseits

22 Eine Erklärung dafür könnte die makroharmonische Beobachtung liefern, dass die Dominantseptakkorde am Ende der Sequenzen ( $\mathrm{E}^{7}$ in Takt 3, $\mathrm{G}^{7}$ in Takt 7, $\mathrm{H}^{7}$ in Takt 11) implizit auf die Dreiklangstöne der Grundtonart a-Moll verweisen und so über die gesamte Passage hinweg ein harmonisches Gravitationszentrum etabliert wird, ohne dass die Tonika selbst erklingt (vgl. Kurth 1920, 320f.; Mitchell 1985, 244f.; Danuser 1998, 835f.).

23 Karsten 1951, 296.

24 Poos 1971, 541.

25 Ünlü 2003, 5.

26 Zu den Eingangstakten der Tristan-Einleitung als Sequenz vgl. neben Poos 1971 und 1987 sowie Ünlü 2003 auch Fladt 2009 und Rohringer 2018. 
der Einflüsse satztechnischer Traditionen und der Hierarchisierung von Akkordtönen beobachtbar macht. ${ }^{27}$

\section{Literatur}

Bailey, Robert (Hg.) (1985), Wagner: Prelude and Transfiguration from »Tristan and Isolde«. The Authoritative Scores, Historical Background, Sketches and Drafts, Views and Comments, Analytical Essays, New York: Norton \& Company.

Berger, Karol (2007), Bach's Cycle, Mozart's Arrow. An Essay on the Origins of Musical Modernity, Berkeley (CA): University of California Press.

Cohn, Richard (2012), Audacious Euphony. Chromaticism and the Triad's Second Nature, New York: Oxford University Press.

Danuser, Hermann (1998), „Tristanakkord", in: Die Musik in Geschichte und Gegenwart, 2. Aufl., hg. von Ludwig Finscher, Sachteil Bd. 9, Kassel: Bärenreiter / Stuttgart: Metzler, 831-844.

Douthett, Jack / Peter Steinbach (1998), »Parsimonious Graphs: A Study in Parsimony, Contextual Transformations and Modes of Limited Transposition", Journal of Music Theory 42/2, 241-263.

Fladt, Hartmut (2009), »Modell - Topos - Figur: Individualisierte Satztechniken in Wagners `Tristan und Isolde««, in: Systeme der Musiktheorie, hg. von Clemens Kühn und John Leigh, Dresden: Sandstein, 20-32.

Harrison, Daniel (1994), Harmonic Function in Chromatic Music: A Renewed Dualist Theory and an Account of its Precedents, Chicago: University of Chicago Press.

Hepokoski, James / Warren Darcy (2006), Elements of Sonata Theory. Norms, Types, and Deformations in the Late-Eighteenth-Century Sonata, New York: Oxford University Press.

Karsten, Werner (1951), »Harmonische Analyse des Tristan-Akkordes«, Schweizerische Musikzeitung 91, 291-296.

Kurth, Ernst (1920), Romantische Harmonik und ihre Krise in Wagners 'Tristanı, Berlin: Hesse, Reprint Hildesheim: Olms 1985.

Mitchell, William J. (1985), »The Tristan Prelude. Techniques and Structure« [1967], in: Wagner: Prelude and Transfiguration from »Tristan and Isolde». The Authoritative Scores, Historical Background, Sketches and Drafts, Views and Comments, Analytical Essays, hg. von Robert Bailey, New York: Norton \& Company, 242-267.

Morgan, Robert P. (2000), „Circular Form in the sTristan« Prelude«, Journal of the American Musicological Society 35/1, 69-103.

Poos, Heinrich (1971), »Der dritte Tristanakkord: Zur Harmoniestruktur der Takte 1-16 der Einleitung von `Tristan und Isolde«", in: Bericht über den internationalen Musikwissenschaftlichen Kongress Bonn 1970, hg. von Carl Dahlhaus und Hans Heinrich Eggebrecht, Kassel: Bärenreiter, 539-542.

27 Wir danken Christian Thorau und der Jury des wissenschaftlichen Wettbewerbs 2019 der Gesellschaft für Musiktheorie für ihre kritischen und konstruktiven Kommentare zu einer früheren Version dieses Artikels. 
Poos, Heinrich (1987), »Die `Tristan«-Hieroglyphe: Ein allegoretischer Versuch«, in: Richard Wagner: Tristan und Isolde, hg. von Heinz-Klaus Metzger und Rainer Riehn, München: edition text + kritik, 46-103.

Rieke, Jakob (2019), „Cycling in Tonal Space. Neo-Riemannian Theory in der dritten Dimension«, ZGMTH 16/1, 41-65. https://doi.org/10.31751/1009 (15.12.2019)

Rohringer, Stefan (2018), »Form, Satzmodelle und Tonfelder in Richard Wagners Vorspiel zu >Tristan und Isolde«", Musiktheorie 33/1, 63-86.

Rudolph, Pascal / Mats Küssner (2018), „Visual Figures of Musical Form between Musicological Examination and Auditory Perception based on Morgan's Analysis of the 'Tristan< Prelude«, Music \& Science 1, 1-10. https://doi.org/10.1177/2059204318794364 (15.12.2019)

Taylor, Benedict (2010), „Cyclic Form, Time, and Memory in Mendelssohn's A-Minor Quartet, Op. 13«, The Musical Quarterly 93/1, 45-89.

Taylor, Benedict (2016), The Melody of Time. Music and Temporality in the Romantic Era, New York: Oxford University Press.

Tymoczko, Dmitri (2011), A Geometry of Music, New York: Oxford University Press.

Ünlü, Altug (2003), »Der `Tristan-Akkord` im Kontext einer tradierten Sequenzformel «, Musiktheorie 2/1, 1-7.

Voss, Egon (Hg.) (2013), Richard Wagner. Tristan und Isolde. Klavierauszug nach der Gesamtausgabe. WWV 90, Mainz: Schott.

Jabs, Ansgar / Rudolph, Pascal (2019): Visualisierung harmonischer Prozesse mithilfe des Circular Pitch-Class Space am Beispiel der Tristan-Sequenz [Visualization of Harmonic Processes by Circular Pitch-Class Space Using the Example of the "Tristan" Sequence]. ZGMTH 16/2, 167-177.

https://doi.org/10.31751/1016

(C) 2019 Ansgar Jabs (ansgar.jabs@gmx.de), Pascal Rudolph (prudolph@uni-potsdam.de)

Universität Potsdam, Universität Potsdam

Dieser Text erscheint im Open Access und ist lizenziert unter einer

Creative Commons Namensnennung 4.0 International Lizenz.

This is an open access article licensed under a

(c)

Creative Commons Attribution 4.0 International License.

eingereicht / submitted: 03/11/2019

angenommen / accepted: 03/11/2019

veröffentlicht / first published: 23/12/2019

zuletzt geändert / last updated: 12/01/2020 